\title{
Using Advanced Industrial Robotics for Spacecraft Rendezvous and Docking Simulation
}

\author{
Toralf Boge and $\mathrm{Ou} \mathrm{Ma}$
}

\begin{abstract}
One of the most challenging and risky missions for spacecraft is to perform Rendezvous and Docking (RvD) autonomously in space. To ensure a safe and reliable operation, such a mission must be carefully designed and thoroughly verified before a real space mission can be launched. This paper describes a new, robotics-based, hardware-in-the-loop RvD simulation facility which uses two industrial robots to simulate the 6-DOF dynamic maneuvering of the docking satellites. The facility is capable of physically simulating the final approaching within 25-meter range and the entire docking/capturing process in a satellite on-orbit servicing mission. The paper briefly discusses the difficulties of using industrial robots for HIL contact dynamics simulation and how these problems are solved.
\end{abstract}

\section{INTRODUCTION}

$\mathrm{W}$ ith the increasing activities for planetary exploration and satellite on-orbit servicing, space missions requiring physical contact (including low-speed impact) become more common than ever. A critical step for satellite on-orbit servicing is to successfully rendezvous and docking to the target satellite (also called client or serviced satellite) in orbit. Autonomous capture or docking is a very difficult and risky operation and therefore, the docking/capture system of a servicing spacecraft has to be thoroughly tested and verified before a real space mission can be launched. Ground-based test and verification of the dynamic responses of a spacecraft to a general 3-D physical contact in space environment is very difficult. The conventional microgravity test technologies have difficulties in testing full 6-DOF contact dynamics of large and complex space systems. For examples, the parabolic flight can only mimic 20 30 seconds of flight time inside a very limited cargo space, which is inappropriate for testing a complete docking process; the counterweight-balance technology suffers extra inertia effects which becomes significant during a contact motion due to resulting large accelerations; the air-bearing based floating test floor is only a 2-D or pseudo 3-D system and also subjects to extra inertial burden due to the needed massive supporting frame/structure; the water-based neutral buoyancy technique alters the dynamics characteristics of the tested system because of the water drag. Further, it cannot be used to test real space hardware containing electronic hardware due to the water environment. Only does a robotics-based active gravity compensation system have no limits on the complexity of the space system to be simulated

Manuscript received December 20, 2010.

Dr. T. Boge is project leader with the German Aerospace Center (DLR), Wessling, 82234, Germany (Corresponding author, phone: $++49-8153-28$ 2485; fax: ++49-8153-28-1450; e-mail: toralf.boge@ dlr.de).

Dr. O. Ma is a professor with the Department of Mechanical \& Aerospace Engineering, New Mexico State University, Las Cruces, NM 88003, USA (Tel: +1-575-646-6544; e-mail: oma@nmsu.edu). or tested while still retaining a full 6-DOF motion condition. Plus, it can use real physical contact hardware to generate contact forces and thus it is more accurate than any mathematical contact dynamics model used in computerbased simulation. The concept of such a general roboticsbased, contact dynamics simulation facility is illustrated by the diagram shown in Fig. 1. It consists of three basic parts:

1) A real-time satellite simulator used to predict the dynamic responses of the servicing and target satellites based on a multibody dynamics model of the satellites.

2) A 6-DOF robotic system to physically deliver the computer-generated 6-DOF (relative) dynamic motion of the two-satellite system.

3) A mockup of the actual docking hardware, which will make physical contact (docking and capturing) in the simulation to generate contact force and moment.

In the concept of this hardware-in-the-loop (HIL) simulation system, the dynamics of the satellites including the microgravity condition is predicted by a mathematical model of the dynamical system, because it is very difficult to physically produce a full 6-DOF on-orbit dynamic motion of a satellite on the ground but it is rather easy to accurately model and simulate such dynamics on a computer. On the other hand, the contact dynamics is represented by the real hardware contact because such contact action is very difficult to accurately model and simulate on a computer. Therefore, the HIL simulation concept is a hybrid of both math model based software simulation and hardware based physical test. The combined simulation process is intended to take advantages of both.

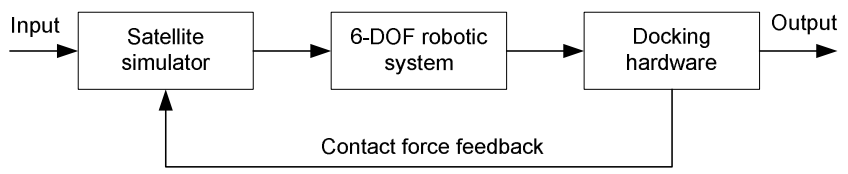

Fig. 1. Three primary parts of an HIL contact dynamics simulator.

In fact, there have been several examples of such simulators for simulating rendezvous and docking operations of space systems. German Aerospace Center (DLR) developed an simulation facility called European Proximity Operations Simulator (EPOS), a former version of the new EPOS facility introduced in this paper, two decades ago for simulating satellite rendezvous operations [1]. The facility was used to support the testing of ATV and HTV rendezvous sensors. NASA/MSF developed an HIL simulator using a 6-DOF Stewart platform for simulating the Space Shuttle being berthed to the International Space Station (ISS) [2, 3]. The Canadian Space Agency (CSA) developed an SPDM Task Verification Facility (STVF) using a giant 6-DOF, customer-built, hydraulic robot to simulate SPDM performing contact tasks on ISS [4, 5]. US Naval Research Lab used two 6-DOF robotic arms to 
simulate satellite rendezvous for HIL testing rendezvous sensors [6]. China is also developing a dual-robot based facility to simulate satellite on orbit servicing operations [7]. The unique features of the new EPOS facility, in comparison with those existing systems, are that it uses two heavypayload industrial robots which can handle a payload up to $250 \mathrm{~kg}$ and it allows one robot to approach the other from 25-meter distance away until zero distance.

\section{OVERVIEW OF THE NEW EPOS FACILITY}

DLR has over two decades of experience of simulating spacecraft RvD operations. The previous EPOS facility (identified as EPOS 1.0) was a testbed jointly developed by ESA and DLR for the simulation of spacecraft maneuvers notably over the last few critical meters of the final rendezvous phase just prior to physical contact. The last intensive utilization of the facility was the test and verification of the ATV sensors and systems which are used for ATV to approach to the ISS [8]. Future applications for satellite on-orbit servicing missions require the facility to be able to provide 6-DOF relative dynamic motion of two satellites in the final approaching phase starting from 25 meters away until the completion of a docking process. The EPOS 1.0 facility could not meet these new requirements and hence, it was replaced by a new EPOS system (identified as EPOS 2.0). The design and construction of the new facility began in 2008 and it has been ready for rendezvous simulation right now. The new facility, as shown in Figs. 2 and 3, is aimed at providing test and verification capabilities for the complete RvD process of a satellite onorbit servicing mission. It comprises a hardware-in-the-loop contact dynamics simulator using two giant industrial robots for physical simulation of full 6-DOF RvD maneuvers.

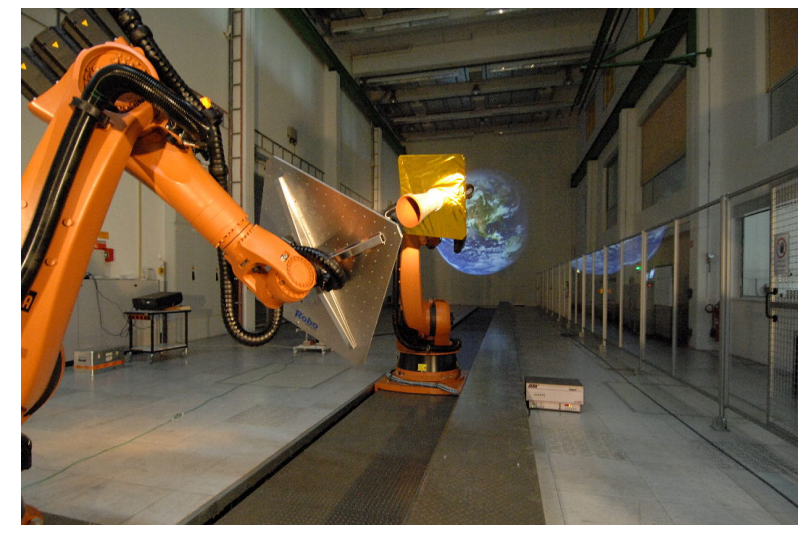

Fig.2 The physical simulation part of the EPOS facility

The main advances of the new EPOS are: 1) It uses two advanced industrial robots which are relatively inexpensive and more reliable in comparison of customer-built robots; 2) it allows one of the robots to move on a 25 -meter long rail system to simulate proximity approaching and rendezvous operation; 3) it simulates sunlight and the visual background conditions; and 4) it has hardware-in-the-loop, zero-gravity, and contact dynamics simulation capabilities allowing highfidelity docking and capturing simulation.

The manipulation specifications of the two industrial robots used by EPOS 2.0 are listed in Table I. Because EPOS will also be used for RvD sensor verification purpose, the facility was extensively calibrated after its installation.
With a laser tracker device, it is confirmed that the facility has achieved an overall positioning accuracy of better than 2 $\mathrm{mm}(3 \mathrm{D} 3 \sigma)$ and an orientation accuracy of $0.2^{\circ}$ (3D $\left.3 \sigma\right)$. In addition, an online measurement system was implemented which measures the relative position between the two robots and commands corrections to the robots. So, the achieved position accuracy will be in sub millimeter range.

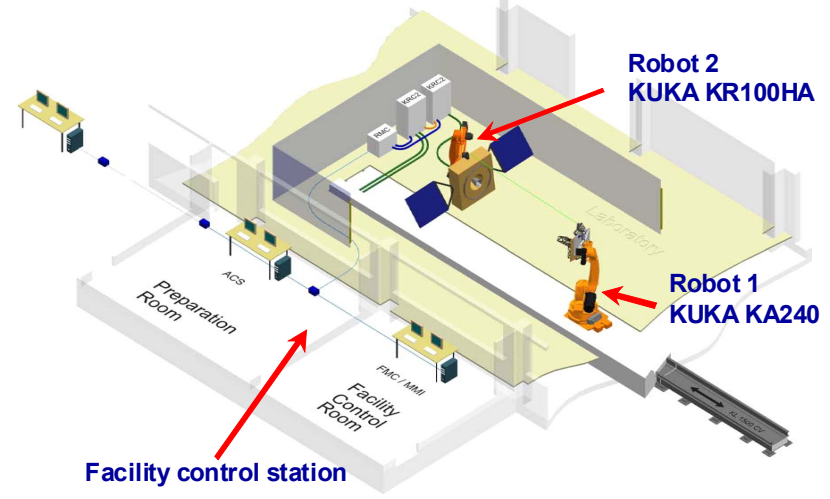

Fig.3 The overview of the EPOS HIL simulation facility

\begin{tabular}{lcc}
\multicolumn{3}{c}{ TABLE I EPOS 'MOTION CAPABILITIES } \\
\hline Parameter & Robot 1 & Robot 2 \\
\hline $\mathrm{X}[\mathrm{m}]$ & $-2,5-+2,5$ & $-2,5-+24,5$ \\
$\mathrm{Y}[\mathrm{m}]$ & $-1,0-+4,0$ & $-2,5-+2,5$ \\
$\mathrm{Z}[\mathrm{m}]$ & $-0,5-+1,5$ & $-0,5-+1,2$ \\
Roll [deg] & $-300-+300$ & $-300-+300$ \\
Pitch [deg] & $-90-+90$ & $-90-+90$ \\
Yaw [deg] & $-90-+90$ & $-90-+90$ \\
\hline Translational [m/s] & 2 & 2 \\
Rotational [deg/s] & 180 & 180 \\
\hline Command rate $[\mathrm{Hz}]$ & 250 & 250 \\
\hline
\end{tabular}

\section{RENDEZVOUS NAVIGATION SIMULATION}

A typical setup of the EPOS facility for a DEOS RvD simulation scenario is shown in Fig.4. For such an operational scenario the $\mathrm{RvD}$ sensors and the space manipulator are mounted on one of the robots and a typical satellite mockup of the target satellite is mounted on the other robot. The RvD sensors can measure the relative position and attitude of the target satellite and the onboard computer calculates on this basis the necessary thrusters or reaction wheel commands. These will be fed into a real-time simulator. This dynamics simulator computes the state vector (position and attitude of the spacecraft) of the next sample update based on relevant environmental and control forces and torques. Then the computed new state vector will be used as the new command to drive the facility.

One important item of the simulation system is the processing of the images coming from the navigation cameras. There are two different approaches being implemented to serve two different distance ranges. First, there are methods for tracking the target in close range, providing full 6-DOF information. Second, there are also methods for tracking the target at a far distance. In this case, however, only the direction to the target and its approximate distance can be estimated. 


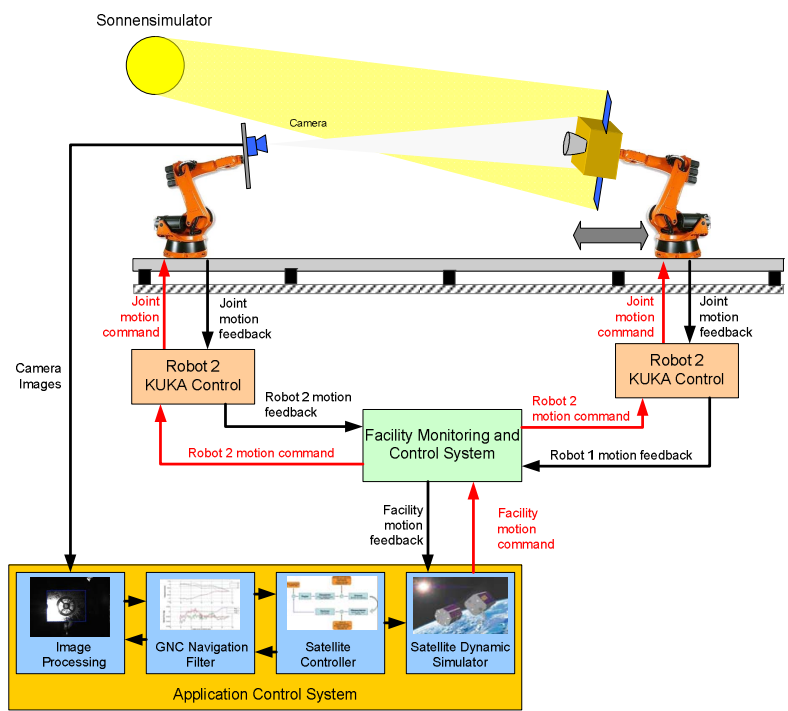

Fig. 4. EPOS HIL simulation scenario

The close-range method is based on a hybrid edge tracker, which relies on classic edge tracking using the ADM detector [10] in situations where this algorithm provides stable results. In cases of low contrast, where all classic edge tracking algorithms fail, a more recent method is used: texture segmentation. The choice of algorithm depends on the confidence indicator given by both algorithms. In practice, this provides a quite stable estimate of outer edges of the target object. Combined with knowledge about its size, sufficient information is available to estimate the full 6DOF pose of the target object. This method, however, needs a very accurate model and the object itself needs to be of a shape which provides a sufficient number of edges to detect. It will also work with cylindrical objects as long as there is some surface texture which allows estimating the orientation. Details of this method can be found in [10].

\section{CONTACT DYNAMIC SIMULATION}

The architecture of the high-level EPOS control system is illustrated in the diagram shown in Fig.5. The two robots are controlled to strictly track the computer simulated satellite motion trajectory. When a physical contact happens, the contact force and moment generated by the docking hardware will be fed back to the satellite simulator. As a result, the simulated satellite motion will be affected (with a time delay). For such a HIL simulation system to have high simulation fidelity, it is required that the simulated docking behavior (which is measured by the resulting motion state and contact force-mention) must be the same as that of the real satellites in space. Such a fundamental requirement cannot be met without the following two conditions:

(1) The two ground robots used to deliver the simulated satellites' relative motion shall respond the HIL control command very fast.

(2) When reacting to a physical contact during a docking operation, the robots (at their tip) shall dynamically behave like the on-orbit satellites being simulated. This is equivalent to impedance matching.

Although the necessity of the requirement (1) is not difficult to understand, it is not easy to meet for the EPOS system because the two KUKA robots are industrial robots with massive bodies. They were designed for usual industrial applications, such as working in an automobile assembly line, and thus, the robots do not have very fast responding speed. The known responding time of the robots is about 4-8 command cycles and each command cycle takes about 4 milliseconds [11]. In other words, the duration from the time when the EPOS control system issues a control command to the time when the robot physically reacts to the command can be up to 8 command cycles or $32 \mathrm{~ms}$. This is a large time delay for controlling a robot to perform contact motion. Moreover, the maximum sampling rate of the robots is the same as their commanding rate, $250 \mathrm{~Hz}$. Such a rate has been quite high for a usual industrial robot but it is not considered high for a robot to be used for the HIL contact dynamics simulation. A preferred sample rate shall be at least $1000 \mathrm{~Hz}$ [5]. Since the robots must be used as is in the EPOS system, a special process control must be developed to handle the time delay problem. The Robotics and Mechatronics Institute of DLR is currently developing technology to practically solve the problem. Their approach is based on a principle of actively balancing the energy inputting to and that outputting from the robotic system. In other words, the method is trying to achieve a passivity behavior of the industrial robots in the HIL simulation process, so that the simulation instability due to the time delay will not happen. Details of the approach will be described in a separate paper in the near future.

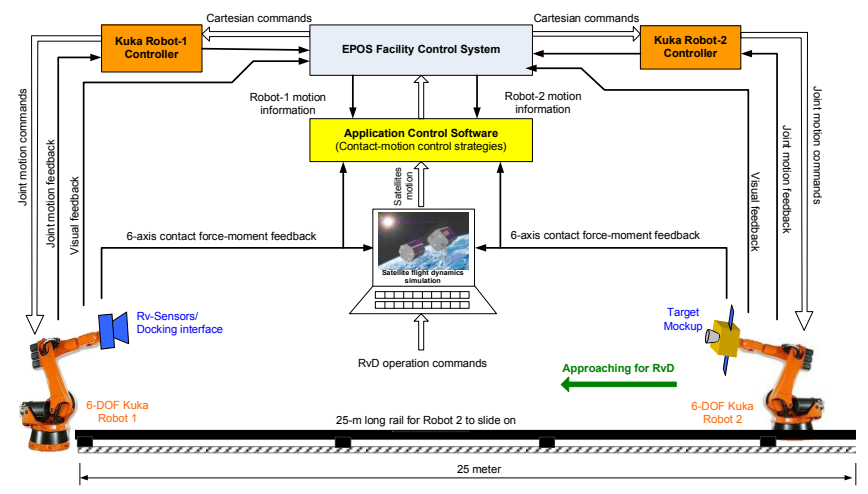

Fig. 5 Control system of the EPOS facility

Obviously, the requirement (2) cannot be met by the industrial robots as is either because the robots are basically positioning machines with high stiffness. Therefore, when the robot's tip is in physical contact with an external object during docking, it may not comply with the resulting contact force as a free-floating satellite does. The robot may even encounter instability in a stiff contact case. Note that whether a contact operation to be simulated is stiff or not should solely depend on the satellites and their docking interfaces rather than the two industrial robots. Therefore, a control loop outside the industrial robots needs to be implemented to deal with this problem (since no inner control loops of the industrial robots can be accessed and modified). An end-effort force control method cannot be used here because the reference contact force for a proper docking operation can never be known in advance. An ideal approach would be to apply an impedance control strategy [13]. However, this requires torque control capability at the joint level, which is not available in the KUKA robots. All we have from the robots is an end-effector position or rate control capability. Similarly, many other advanced and 
proven robot control strategies, such as the computed torque control [14], passivity-based control [15], etc. cannot be implemented either because the robots either do not have a joint torque control capability or do not have an inverse dynamics model (robot manufacturers usually do not provide dynamics models of their products). As a result, an endeffector admittance control strategy is employed to deal with the problem because such a control strategy does not need a joint torque control capability nor a dynamics model of the robot for implementation but it still can achieve the required impedance behaviour at the tip during a contact operation. The controller, shown in Fig.6, is briefly described next.

The dynamics of the servicing satellite can be described by the following governing equation:

$$
\mathbf{f}_{s}=\mathbf{M}\left(\Delta \mathbf{x}_{s}\right) \Delta \ddot{\mathbf{x}}_{s}+\mathbf{C}\left(\Delta \mathbf{x}_{s}, \Delta \dot{\mathbf{x}}_{s}\right) \Delta \dot{\mathbf{x}}_{s}+\mathbf{K}\left(\Delta \mathbf{x}_{s}\right) \Delta \mathbf{x}_{s}
$$

where $\mathbf{f}_{s} \in R^{6}$ is the resultant external force-moment of the satellite; $\mathbf{M} \in R^{6 \times 6}$ is the inertia matrix of the dynamical system; $\mathbf{C} \in R^{6 \times 6}$ is the damping matrix; $\mathbf{K} \in R^{6 \times 6}$ is the stiff matrix; $\Delta \mathbf{x}_{s} \in R^{6}$ is the relative position and orientation of the satellite with respect to the target satellite. Since only the relative motion is of interest, the dynamics equations of the target satellite reduce to the Euler equations only. If both satellites are known, these equations are also known.

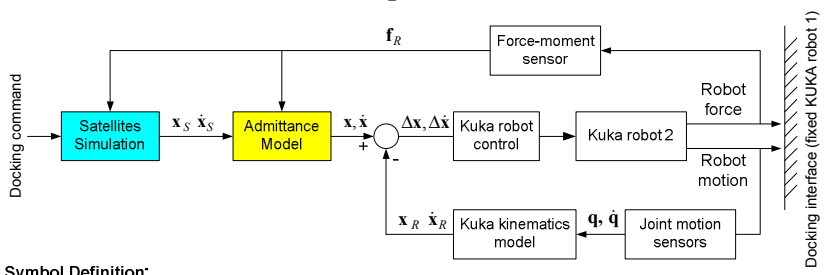

Symbol Definition:

$\mathbf{x}_{S} \dot{\mathbf{x}}_{S}, \ddot{\mathbf{x}}_{S}$-desired Cartesian position, velocity and acceleration
$\mathbf{x}_{R}$ - $\mathbf{x}_{R}$ $x, \mathbf{x}$-Desired Cartesian position and velocity determined by the $\mathbf{f}_{\mathrm{R}}-$ Actual contact force-moment in Cartesian space
$\mathbf{q}, \dot{\mathbf{q}}-$ Actual joint positions and rates of the robot

Fig. 6 Admittance control strategy for EPOS robot control

For admittance control of the EPOS robots, we need to assume an impedance model of the robotic system in terms of the relative motion between the two robots, namely,

$$
\mathbf{f}_{r}=\mathbf{M}_{r} \Delta \ddot{\mathbf{x}}_{r}+\mathbf{C}_{r} \Delta \dot{\mathbf{x}}_{r}+\mathbf{K}_{r} \Delta \mathbf{x}_{r}
$$

where $\mathbf{f}_{r} \in R^{6}$ is the resultant contact force-moment exerted on the tip of the approaching robot. Since the robot is controlled to simulate the dynamic behavior of the docking satellite. It is reasonable that the coefficient matrices of the impedance model (2) are chosen to be the same as those given in the satellite dynamics model (1). In other words, the admittance control is intended to make the industrial robot behave at its tip like the servicing satellite during a docking operation. Thus, the admittance control law is chosen as

$$
\Delta X=\left(\mathbf{M} s^{2}+\mathbf{C s}+\mathbf{K}\right)^{-1} F
$$

where $\Delta X$ and $F$ are the Laplace transforms of the relative position-orientation and the contact force-moment between the two robots, respectively. Contact force $\mathbf{f}_{r}$ is measured by a 6-axis force-moment sensor installed behind the docking interface hardware. For simplification, the coefficient matrices of the impedance model (2) may be assumed constant. This approximation is valid because the relative docking speeds of all the known real space docking cases are very low (no more than several centimeters per second).

The reference position value obtained from (3) is only a desired end-effector trajectory for the robot control. The robot may not be able to reach the desired trajectory because of inevitable disturbances and uncertainties. To make sure that the robot can accurately track a desired trajectory, an end-effector position feedback loop should be added for improving the robot's tracking performance.

\section{CONCLUSIONS}

A new, robotics-based hardware-in-the-loop (HIL) rendezvous and docking simulation facility developed by DLR was introduced. The facility was designed for system level test and verification of proximity-rendezvous and docking operations of satellite on-orbit servicing missions. Comparing with the few existing robotics-based HIL docking simulation systems currently being used by the major space agencies around the world, this facility is unique for: 1) it uses two industrial robots (instead of customer-built robots); 2) it allows testing rendezvous within last 25-meter distance; 3) it simulates representative sunlight and visual background conditions; 4) it has HIL contact dynamics simulation capability allowing high-fidelity docking simulation. The facility has been ready for simulating rendezvous tasks and will be ready soon for simulating docking operations.

\section{REFERENCES}

[1] R. Krenn and B. Schaefer, "Limitations of hardware-in-the-loop simulations of space robotics dynamics using industrial robots", European Space Agency, ESA SP-440, Aug. 1999, pp. 681-686.

[2] S. Ananthakrishnan, R. Teders, and K. Alder, "Role of estimation in real-time contact dynamics enhancement of space station engineering facility", IEEE Rob. and Auto. Mag., Vol. 3(3), 1996, pp. 20-28.

[3] F.D. Roe, R.T. Howard, and L. Murphy, "Automated rendezvous and capture system development and simulation for NASA", Proc. SPIE, Vol. 5420, 118 (2004); doi:10.1117/12.542529.

[4] J.-C. Piedboeuf, J. De Carufel, F. Aghili, and E. Dupuis, Task verification facility for the Canadian special purpose dextrous manipulator, IEEE Int Conf on Rob and Auto, Detroit, 1999, Vol. 3, pp. 1077-1083.

[5] O. Ma, J. Wang, S. Misra, M. Liu, "On the validation of SPDM task verification facility”, J. of Rob. Syst., Vol.21(5), 2004, pp. 219-235.

[6] R. Bell et al., "Hardware-in-the-loop tests of an autonomous gn\&c system for on-orbit servicing", AIAA-LA Section/SSTC Responsive Space Conference 2003.

[7] W. F. Xu, B. Liang, Y. S. Xu, C. Li and W. Y. Qiang, "A Ground Experiment System of Free-floating Space Robot for Capturing Space Target", J. of Intell. and Rob. Syst., Vol.48(2), pp. 187-208, 2007.

[8] T. Boge et. al. Hardware-In-The-Loop Simulator für Rendezvous- und Dockingmanöver. In Proceedings of DGLR-conference, 2009.

[9] T. Rupp, T. Boge, R. Kiehling, and F. Sellmaier. "Flight dynamics challenges of the german on-orbit servicing mission DEOS". Proc. 21th International Symposium on Space Flight Dynamics, 2009.

[10] T. Tzschichholz and T. Boge; "GNC Systems Development in Conjunction with a RVD Hardware-in-the-loop Simulator"; 4th Intl Conf. on Astrodynamics Tools and Tech.; Madrid, 3-6 May 2010.

[11] EPOS Facility manual. Robo-Technology GmbH.

[12] B. Shaefer, R. Krenn, M. Loesch, and W. Rulka, "Space robotics dynamics simulation performance in real-time environments", $A d v$. in Comp. Multibody Dyn, Lisbon, Portugal, Sept.20-23, 1999.

[13] N. Hogan, "Impedance Control: An Approach to Manipulation", Proc. of American Control Conference, 1984, pp.304 - 313.

[14] R. H. Middleton and G. C. Goodwin, "Adaptive computed torque control for rigid link manipulations", Syst. \& Ctrl. Letters, Vol. 10(1), January 1988, pp.9-16.R.

[15] O.A.J. van der Schaft, I. Mareels, and B. Maschke, "Putting energy back in control”, IEEE Ctrl. Syst. Mag., February 2001. 